\title{
Gráficos difusos versus gráficos tradicionales para el control de procesos por atributos
}

\section{Vivian Lorena Chud Pantoja, ${ }^{1 *}$ Nathaly Martínez Escobar ${ }^{2}$ Juan Carlos Osorio Gómez ${ }^{3}$}

\author{
' Universidad Santiago de Cali, Facultad de Ingeniería, Departamento Gestión de Tecnología, Ingeniería de Operaciones y Medio Ambiente, Grupo de Investi- \\ gación IDEAS, calle 5 n. ${ }^{\circ}$ 62-00. Santiago de Cali, Colombia. \\ * Autor para correspondencia: vivianchud@usc.edu.co \\ http://orcid.org/0000-0001-8842-637X \\ 2 Universidad Santiago de Cali, Facultad de Ingeniería, Departamento Gestión de Tecnología, Ingeniería de Operaciones y Medio Ambiente, calle 5 n. $62-00$. \\ Santiago de Cali, Colombia. \\ http://orcid.org/0000-0001-8842-637X
}

${ }^{3}$ Universidad del Valle, Escuela de Ingeniería Industrial, Grupo de Investigación en Logística y Producción, calle 13 n. $100-00$. Santiago de Cali, Colombia. https://orcid.org/0000-0001-5625-5609

\section{RESUMEN}

Los gráficos de control son una buena herramienta para controlar procesos. Han sido ampliamente utilizados -y aún ahora se utilizan - en la mayoría de los procesos manufactureros. Sin embargo, se han presentado propuestas orientadas a mejorar el desempeño de los mismos, principalmente en los aspectos referentes a la incertidumbre y ambigüedad existente en los datos. En este sentido, los gráficos de control difusos son una alternativa valiosa para mejorar el desempeño de los gráficos tradicionales. Se presenta, entonces, una comparación de los gráficos de control Shewhart (tradicionales) y los gráficos de control difusos por atributos, con el objetivo de establecer las similitudes y diferencias existentes entre las dos metodologías. De esta manera, se desarrolla un ejemplo numérico de un gráfico tradicional c y gráficos difusos construidos a partir de las siguientes técnicas de transformación: moda difusa, mediana difusa, rango medio difuso y enfoque difuso directo. Para realizar una comparación se utilizaron las reglas de evaluación de patrones de comportamiento no natural en un gráfico de control. A partir de los resultados de la comparación realizada en esta investigación se concluye que al utilizar las reglas de evaluación en ambos gráficos con los mismos datos no se obtienen diferencias en los resultados.

Palabras clave: gráficos de control, control de proceso, datos difusos, gráficos de control difusos.

\section{Diffuse vs. Traditional Graphics for Control of Processes by Attributes}

Citation: Chud Pantoja, V. L. \& Martínez Escobar, N. \& Osorio Gómez J.C. (2017). Gráficos difusos versus gráficos tradicionales para el control de procesos por atributos. Mutis 7(1), 34-47, doi: http://dx.doi. org/10.21789/22561498.1189

Received: Abril 12, 2016. Accepted: Febrero 15, 2017. Published on line: Mayo 1, 2017.

Copyright: ( 2016 Chud Pantoja, V. L. \& Martínez Escobar, N. \& Osorio Gómez J. C. This is an open-access article, which permits unrestricted use, distributions and reproduction in any medium, provided the original author and source are credited.

Competing Interests: The authors have no conflict of interest.

\begin{abstract}
Control charts are the main tool in the process control. They have been widely used and are still used in most manufacturing processes. However, proposals have emerged that seek to improve their performance, mainly about topics which involve the vagueness and uncertainty of the data. In this sense, fuzzy control charts area an important alternative for improve the performance of control charts. We present a
\end{abstract}


comparison of Shewhart control charts (traditional) and fuzzy control charts for attributes with the aim of establishing similarities and differences between the two methodologies. We develop a numerical example for a c traditional chart and fuzzy charts these built with different methods: Fuzzy mode, fuzzy median and fuzzy midrange and the direct fuzzy approach, then we do the comparison used the rules for evaluation of patterns of not natural behavior in the control charts. From the results of the comparison carried out in this research, it is concluded that when using the evaluation rules for both graphs with the data used, no differences are obtained in the results.

Keywords: Control charts, process control, fuzzy data, fuzzy control charts.

\section{INTRODUCCIÓN Y REVISIÓN DE LA LITERATURA}

Si bien las técnicas de control de proceso han venido desarrollándose desde principios del siglo pasado, las organizaciones actuales requieren controlar sus procesos para garantizar la calidad de sus resultados. De ahí que el control estadístico de proceso (SPC, por sus siglas en inglés) sea una actividad vigente en la industria moderna. Tal como afirman Evans y Lindsay
(2005), el SPC se apoya en las gráficas de control debido a que son las herramientas básicas de mejora de calidad.

Los gráficos de control son ampliamente usados en la práctica de producción, en procesos en los requerimientos de calidad y los datos de calidad son definidos usualmente con precisión (Hryniewicz, 2007).

Las variaciones de un proceso pueden ser aleatorias (naturales o comunes, y son inherentes al proceso), y especiales (o asignables), en las que las variaciones son irregulares. Lo ideal es que solo existan causas aleatorias en el proceso, de modo que se pueda decir que este se encuentra bajo control. Como afirman Gryna, Chua y Defeo (2007), "un proceso que opera sin causas especiales de variación está en un estado de control estadístico"; de allí que uno de los principales objetivos de un gráfico de control sea detectar las causas especiales (o asignables) de variación en un proceso, mediante el análisis de datos (Gryna et al., 2007). Es necesario analizar el proceso cuando la variación excede los límites de control, pues esto indica que existen causas especiales en el proceso. Tanto Gryna et al. (2007) como Evans y Lindsay (2005) presentan algunos pasos generales para realizar los gráficos de control, los cuales se tomaron como base para elaborar la tabla 1.

Tabla 1. Pasos requeridos para el desarrollo y utilización de un gráfico de control

\begin{tabular}{|c|c|c|}
\hline 1. & $\begin{array}{l}\text { Elegir las características } \\
\text { que se van a diagramar }\end{array}$ & $\begin{array}{l}\text { - } \quad \text { Escoger la variable o atributo que se va a medir } \\
\text { - } \quad \text { Determinar la base, el tamaño y la frecuencia de la muestra }\end{array}$ \\
\hline 2. & $\begin{array}{l}\text { Elegir el tipo de gráfico } \\
\text { de control }\end{array}$ & $\begin{array}{l}\text { - Seleccionar entre los tipos de gráficos de control tradicionales existentes los que se van } \\
\text { a utilizar }\end{array}$ \\
\hline 3. & $\begin{array}{l}\text { Determinar los límites de } \\
\text { control de prueba }\end{array}$ & $\begin{array}{l}\text { - Decidir sobre la línea central que se usará y la base para el cálculo de los límites. La línea } \\
\text { central puede ser el promedio de los datos anteriores o un promedio deseado, en caso } \\
\text { de que se esté construyendo la información del proceso por primera vez }\end{array}$ \\
\hline 4. & $\begin{array}{l}\text { Elegir el "subgrupo } \\
\text { racional" (o } \\
\text { muestra) }\end{array}$ & - De acuerdo con las características particulares del proceso \\
\hline 5. & Recolectar los datos & $\begin{array}{ll}\text { - } & \text { Registrar los datos } \\
\text { - } \quad \text { Calcular estadísticas relevantes: promedios, rangos, proporciones, etc. } \\
\text { - } \quad \text { Trazar los datos estadísticos sobre la gráfica }\end{array}$ \\
\hline 6. & $\begin{array}{l}\text { Calcular los límites de } \\
\text { control }\end{array}$ & $\begin{array}{l}\text { - Dibujar la línea central (promedio del proceso) sobre la gráfica } \\
\text { - Calcular los límites de control superior e inferior }\end{array}$ \\
\hline 7. & Analizar e interpretar & $\begin{array}{ll}\text { - } & \text { Investigar la gráfica para buscar si hay falta de control } \\
\text { - } \quad \text { Eliminar puntos fuera de control, si es necesario } \\
\text { - } \quad \text { Volver a calcular los límites de control, si es necesario }\end{array}$ \\
\hline 8. & $\begin{array}{l}\text { Utilizar como } \\
\text { herramienta para } \\
\text { la solución de los } \\
\text { problemas }\end{array}$ & $\begin{array}{l}\text { - } \quad \text { Continuar con la recolección de datos y con el trazado } \\
\text { - Identificar situaciones fuera de control y tomar acciones correctivas }\end{array}$ \\
\hline
\end{tabular}


Los gráficos de control tradicionales se dividen en dos grandes grupos: gráficos de control por variables y por atributos. Los gráficos de control por atributos están basados en la observación de la presencia o ausencia de una determinada característica, o de cualquier tipo de defecto en el producto; es decir, en esta clase de gráficos se supone una clasificación dicotómica para la muestra (bueno o malo, conforme o no conforme, $\mathrm{u}$ otro). Asimismo, son utilizados para contrastar características cualitativas. Los gráficos de control por atributos se clasifican en

- Gráfico p: proporción de defectuosos o fracciones no conformes

- Gráfico $n p$ : número de defectuosos

- Gráfico c: número de defectos

- Gráfico u: defectos por unidad).

En este documento se desarrollará el gráfico de control $c$.

Por otra parte, dado que el mundo es impreciso, que los actos de los hombres y las relaciones que establecen entre sí están afectados de vaguedad, la realidad no puede estudiarse en términos absolutos con técnicas aplicables a situaciones ciertas ni aleatorias, determinando un pertenece o no pertenece, como en la teoría de conjuntos clásicos (Lazzari, Machado y Pérez; 1998); por ello se ha desarrollado la lógica difusa.

Con el fin de mejorar los resultados derivados del control de procesos e incluir en el análisis los datos vagos e imprecisos, se proponen los gráficos de control difusos, que introducen elementos de la lógica difusa en los gráficos tradicionales, o de Shewhart. La lógica difusa permite construir una estructura matemática con la cual es posible manipular datos inciertos o vagos, para los cuales la pertenencia a un conjunto tiene grados entre el pertenece y no pertenece, entre verdadero y falso; en otras palabras, esta puede tomar un valor real del intervalo [0.1]. Esta lógica busca describir y formalizar la realidad empleando modelos flexibles que interpreten el comportamiento humano y las relaciones entre los hombres (Lazzari et al., 1998). La lógica difusa permite expresar el conocimiento de forma más natural, lo cual hace que muchos problemas de ingeniería y de toma de decisiones se simplifiquen significativamente (Briceño, 2004).

Mediante la unión de los gráficos de control tradicionales y la lógica difusa se obtienen los gráficos de control difusos. Estos también se clasifican en gráficos por variables y por atributos (Gülbay y Kahraman, 2007).
En los gráficos de control, los juicios clásicos se basan en clasificaciones binarias como "en control" o "fuera de control". En los gráficos de control difusos, las decisiones intermedias, tales como "más bien en control" o "más bien fuera de control", pueden ser manipuladas con el fin de convertir términos lingüísticos, que en los gráficos de control clásicos no pueden ser aplicados fácilmente (Noori, Bagherpour y Zareei, 2008).

Si bien el tema de los gráficos difusos es mucho más reciente que el de los gráficos tradicionales, en la literatura hay una cantidad significativa de trabajos, de los cuales es importante mencionar los siguientes:

Cheng (2005) desarrolla la construcción de gráficos de control con números difusos para procesos cuyos resultados sean tasas de calidad obtenidas a partir de juicios subjetivos de expertos. Por su parte, Hsieh (2005) realizó un análisis para la construcción de gráficos de control difusos por variables teniendo en cuenta números difusos triangulares y trapezoidales, tanto simétricos como asimétricos.

Con respecto a gráficos por variables, se pueden mencionar los trabajos de Senturk y Erginel (2009), quienes presentan una propuesta para la construcción de gráficos de control difusos X-R y X-S utilizando la técnica de los $\alpha$ cortes. Ellos concluyen que con esta técnica la flexibilidad de los límites de control tradicionales se incrementa, y con ella, la efectividad de los gráficos para el control del proceso. Por otra parte, Faraz y Shapiro (2010) desarrollan una propuesta de gráfico de control $\mathrm{X}-\mathrm{S}^{2}$ que, mediante la técnica fuzzy, busca explicar la vaguedad existente en las observaciones. Ellos realizan los gráficos utilizando los diferentes métodos de transformación: rango medio fuzzy, media fuzzy, mediana fuzzy y moda fuzzy. Asimismo, Shu y Wu (2011) proponen un gráfico de control X-R controlar el promedio del proceso y su variación ante la presencia de datos difusos.

Con respecto a gráficos por atributos, cabe mencionar el trabajo de Amirzadeh, Mashinchi y Parchami (2009), quienes proponen un gráfico de control difuso p para controlar un proceso, asumiendo como característica difusa $p$ el grado de no conformidad de la variable, con lo cual logran disminuir el error tipo II en el proceso. 
En los trabajos anteriores se usaban técnicas de defuzzyficación para el manejo final de los datos difusos. Sin embargo, Gülbay y Kahraman (2007) presentan un enfoque difuso directo (DFA, por sus siglas en inglés) para desarrollar los gráficos de control difusos por atributos. A diferencia de los métodos de transformación difusa utilizados, este enfoque no realiza transformación de los datos difusos; de esta manera previene la pérdida de información incluida en las muestras. Estos autores también presentan una propuesta para utilizar las reglas de evaluación de gráficos de control difusos por atributos que permiten evaluar patrones no naturales con base en las reglas de evaluación de gráficos de control tradicionales, definidas usando las probabilidades de eventos difusos. Estas se pueden aplicar con los resultados del DFA (Gülbay y Kahraman, 2006).

Otros trabajos importantes que merecen mención son el de Demirli y Vijayakumar (2010), quienes desarrollaron un sistema de inferencia fuzzy para la identificación de las causas a las que se las puede responsabilizar de variaciones cuando el proceso se encuentra fuera de control. Alaeddini, Ghazanfari y Nayeri (2009) proponen un sistema híbrido que reúne elementos difusos y elementos estadísticos (análisis de clusters) para la identificación del momento real en el cual el proceso sufre un cambio (no cuando es detectado por el gráfico de control). En esta misma línea, y buscando refinar más el proceso, Zarandi y Alaeddini (2010) proponen una metodología similar, pero solamente para gráficos de control con muestras variables.

Finalmente, se pueden encontrar trabajos relacionados con el análisis de capacidad de proceso, como el de Kaya y Kahraman (2011a), en el que proponen el análisis de capacidad de proceso a partir de la construcción de los gráficos de control difusos, específicamente de un gráfico $X-R$, tanto con números triangulares como trapezoidales. Asimismo, ellos plantean una propuesta adicional para el cálculo de la capacidad del proceso cuando los límites de tolerancia no son simétricos (Kaya y Kahraman, 2011b).

En este artículo se realiza una comparación de los gráficos de control tradicionales y los gráficos de control difusos por atributos utilizando reglas de evaluación de patrones de comportamiento no naturales, que permiten establecer similitudes y diferencias en cuanto a su aplicación en el control de procesos. Inicialmente se presenta la definición de gráficos de con- trol difusos, y se incluye su clasificación e interpretación. Posteriormente se detallan las reglas de evaluación para ambos tipos de gráficos. Luego se presenta el ejemplo sobre el que se aplica la medición del desempeño de los gráficos de control tradicionales y los difusos en la construcción de un gráfico por atributos tipo c. Seguidamente se exponen los resultados y, finalmente, las conclusiones.

\section{GRÁFICOS DE CONTROL DIFUSOS}

Los gráficos de control difusos son herramientas que tienen la capacidad de tratar datos vagos, inciertos y con información incompleta. Al igual que los gráficos de control tradicionales, los difusos cuentan con una línea central $(\widetilde{C} L)$ y límites de control superior $(U \widetilde{C} L)$ e inferior $(L \widetilde{C} L)$. La diferencia es que estos son expresados por números difusos. Un número difuso puede ser triangular o trapezoidal, simétrico o asimétri$c o$, representado por $(a, b, d)-(a, c, d)$ y $(a, b, c, d)$, respectivamente. En Lazzari et al. (1988) se establece que se denomina número difuso al subconjunto borroso de números reales, convexo y normal; este se puede definir en cualquier conjunto referencial totalmente ordenado, como, por ejemplo, $\mathrm{R}$ (números reales), Z (números enteros), $\mathrm{N}$ (números naturales). Los números difusos pueden ser trapezoidales o triangulares. Un número difuso trapezoidal $(\bar{N})$ está definido por cuatro números, $\mathrm{a} \leq \mathrm{b} \leq \mathrm{c} \leq \mathrm{d}$, donde el gráfico de $y=\bar{N}(x)$ es un trapezoide con la base del intervalo $[a, d]$ y $\bar{N}(x)=1$ para b $\leq \mathrm{x} \leq \mathrm{c}$ (Csutora y Buckley, 2001); $y=\bar{N}(x)$ es la función de pertenencia. Puede ser simétrico, si $b-a=d-c$, y asimétrico: $(b-a)-(d-c)$ $\neq 0$. Se le denomina número difuso triangular a todo número difuso real, continuo, cuya función de pertenencia determina con el eje horizontal un triángulo. Este queda determinado por tres números reales ( $a$, $b, c)$, tales que $(a \leq b \leq c)$. Puede ser asimétrico si $(b-$ $a)-(c-b) \neq 0$, y simétrico si $(b-a)=(c-b)$. Los números difusos triangulares simétricos (STFNs) se denotan por $\tilde{x}_{i}=\left(a_{i}-d, a_{i}, a_{i}+d\right)$, según Hsieh (2005), y se pueden representar como en la figura 1. 


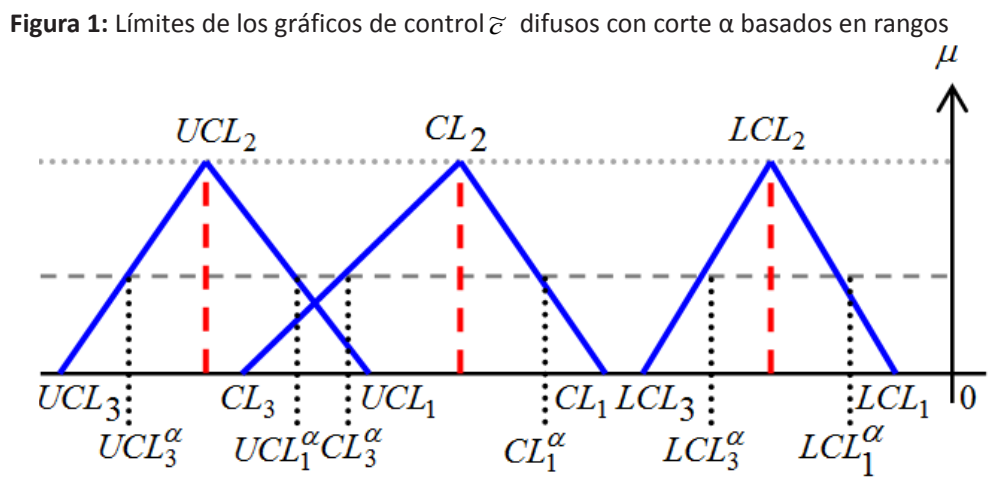

La línea central y los límites de control para un gráfico de control difuso se calculan de la siguiente manera:

$$
\begin{gathered}
\widetilde{C} L=\left[\sum_{j=1}^{m} \frac{a_{j}}{m}, \sum_{j=1}^{m} \frac{b}{m}, \sum_{j=1}^{m} \frac{c}{m_{j}}, \sum_{j=1}^{m} \frac{d}{m}\right]=(\bar{a}, \bar{b}, \bar{c}, \bar{d})=\left(C L_{1}, C L_{2}, C L_{3}, C L_{4}\right) \\
L \widetilde{C} L=\widetilde{C} L-3 \sqrt{\widetilde{C} L}=\left(L C L_{1}, L C L_{2}, L C L_{3}, L C L_{4}\right) \\
U \widetilde{C} L=\widetilde{C} L+3 \sqrt{\widetilde{C} L}=\left(U C L_{1}, U C L_{2}, U C L_{3}, U C L_{4}\right)
\end{gathered}
$$

\section{TÉCNICAS DE TRANSFORMACIÓN FUZZY}

Existen cuatro técnicas de transformación difusa, que son similares a la medida de tendencia central usada por la estadística descriptiva: rango medio difuso de nivel $\alpha$, mediana difusa, promedio difuso y moda difusa (Gülbay y Kahraman, 2007). Adicionalmente, se plantea el método de enfoque difuso directo (DFA) (Gülbay y Kahraman, 2006).

\section{Rango medio difuso de nivel ${ }_{\alpha}$}

El rango medio difuso de nivel $\alpha: f_{m r}^{\alpha}$ para muestras triangulares, se define como el punto medio de los finales de los cortes de nivel $\alpha$. Un corte de nivel $\alpha\left(A^{\alpha}\right)$ es un conjunto no difuso que comprende todos los elementos en los cuales la función de pertenencia es $\geq \alpha$. Si $a^{\alpha}$ y $b^{\alpha}$ son los puntos finales de $A^{\alpha}$, entonces; $f_{m r}^{\alpha}=(1 / 2)\left(a^{\alpha}+b^{\alpha}\right)$, donde $a^{\alpha}=a+\alpha(b-a)$ y $c^{\alpha}=c-\alpha(c-b)$. El rango medio difuso de nivel $\alpha$ de una muestra j, $S_{m r j}^{\alpha}$ se determina por:

$$
S_{m r j}^{\alpha}=\left(\left(a_{j}+c_{j}\right)+\alpha\left[\left(b_{j}-a_{j}\right)-\left(c_{j}-b_{j}\right)\right]\right) / 2
$$

La moda difusa es un caso del rango medio difuso de nivel ${ }^{\alpha}$ cuando $\alpha=1$.

\section{Moda difusa}

La moda difusa de un conjunto difuso $f$ es el valor de la variable base, $X$, donde la función de pertenencia es igual a 1. Esto puede expresarse como

$$
f_{\text {mod }}=\left\{x \in X \mid \mu_{f}(x)=1\right\}
$$

\section{Mediana difusa}

La mediana difusa de nivel $\alpha, f_{\text {med }}^{\alpha}$ es el punto que divide la función de pertenencia de un conjunto difuso en dos regiones iguales en el nivel $\alpha$.

\section{Enfoque difuso directo (DFA [direct fuzzy approach])}

En este enfoque, los datos lingüísticos no se transforman en valores representativos mediante métodos de transformación difusa, con el fin de guardar propiedades representativas de las muestras difusas (Gülbay y Kahraman, 2007). Tanto las muestras como los límites de control están representados y operados como números difusos. Los límites de control difusos de nivel $\alpha$ utilizados en este enfoque se calculan tal como se presenta en las siguientes ecuaciones: 


$$
\begin{gathered}
\widetilde{C} L^{\alpha}=\left(\bar{a}^{\alpha}, \bar{b}, \bar{c}, \bar{d}^{\alpha}\right)=\left(C L^{\alpha}{ }_{1}, C L_{2}, C L_{3}, C L^{\alpha}{ }_{4}\right)(\mathbf{6}) \\
L \widetilde{C} L^{\alpha}=\widetilde{C} L^{\alpha}-3 \sqrt{\widetilde{C} L}{ }^{\alpha}=\left(\bar{a}, \bar{b}, c, \bar{d}^{\alpha}\right)-3 \sqrt{\left(\overline{a^{\alpha}}, \bar{b}, c, \bar{d}^{\alpha}\right)}=\left(L C L^{\alpha}{ }_{1}, L C L_{2}, L C L_{3}, L C L^{\alpha}{ }_{4}\right) \\
U \widetilde{C} L^{\alpha}=\widetilde{C} L^{\alpha}+3 \sqrt{\widetilde{C} L^{\alpha}}=\left(\overline{a^{\alpha}}, \bar{b}, c, \bar{d}^{\alpha}\right)+3 \sqrt{\left(\overline{a^{\alpha}}, \bar{b}, c, \bar{d}^{\alpha}\right)}=\left(U C L^{\alpha}{ }_{1}, U C L_{2}, U C L_{3}, U C L^{\alpha}{ }_{4}\right)
\end{gathered}
$$

La decisión sobre si el proceso está bajo control se puede hacer según el porcentaje del área de la muestra que permanece en el interior de los $L \widetilde{C} L$ y/o $U \widetilde{C} L$. Cuando la muestra está incluida por los límites de control, se dice que el proceso está "en control". Si una muestra difusa está excluida por los límites de control difusos, se dice que el proceso está "fuera de control". De lo contrario, una muestra está parcialmente incluida en los límites de control. En este caso, si el porcentaje del área que está dentro de los límites de control difusos $(\beta)$ es igual o mayor a un porcentaje predefinido $\beta$, entonces el proceso puede ser aceptado como "más o menos en control"; de lo contrario se puede afirmar que está "más o menos fuera de control” (Gülbay y Kahraman, 2006 y 2007).

Los parámetros para la determinación del área de la muestra fuera de los límites de control difusos para el nivel de corte $\alpha$ son $\mathrm{LCL}_{1}, \mathrm{LCL}_{2}, \mathrm{UCL}_{3}, \mathrm{UCl}_{4}$, $a, b, c, d, y \alpha$. La forma de los límites de control difuso y la muestra están formadas por las líneas de
$\overline{\mathrm{LC}} \overline{\mathrm{L}}_{1} \overline{\mathrm{LC}} \overline{\mathrm{L}}_{2} \overline{\mathrm{UCL}}_{3} \overline{\mathrm{UC}} \bar{L}_{4} \bar{a} \bar{b}$ y $\bar{c} \bar{d}$. El diagrama de flujo para calcular el área de la muestra fuera de los límites de control $A_{\text {out }}^{U}$, área de la muestra que cae encima del límite de control superior y $A_{\text {out }}^{L}$, es el área que cae debajo del límite de control inferior) se da en la figura 2, y las ecuaciones para calcular estas áreas se exponen en el anexo.

Entonces, el área total de la muestra fuera de los límites de control difusos es $\left(A_{\text {out }}=A_{\text {out }}^{U}+A_{\text {out }}^{L}\right)$. El porcentaje del área de la muestra dentro de los límites de control se calcula como se indica en la ecuación $\beta_{j}^{\alpha}=\left(S^{\alpha}-A_{\text {out }}^{\alpha}{ }_{j}\right) / S^{\alpha}{ }_{j}$, donde $S^{\alpha}{ }_{j}$ es el área de la muestra en un nivel de $\alpha$-corte. En contraste con los métodos que utilizan las transformaciones difusas, este enfoque es muy flexible y sería más exacto, ya que tanto los datos lingüísticos como los límites de control no se transforman en valores representativos, para evitar la pérdida de las propiedades de las muestras (Gülbay y Kahraman, 2007).

Figura 2. Diagrama de flujo para calcular el área de una muestra difusa (a, b, c, d) que queda fuera de los límites de control difusos

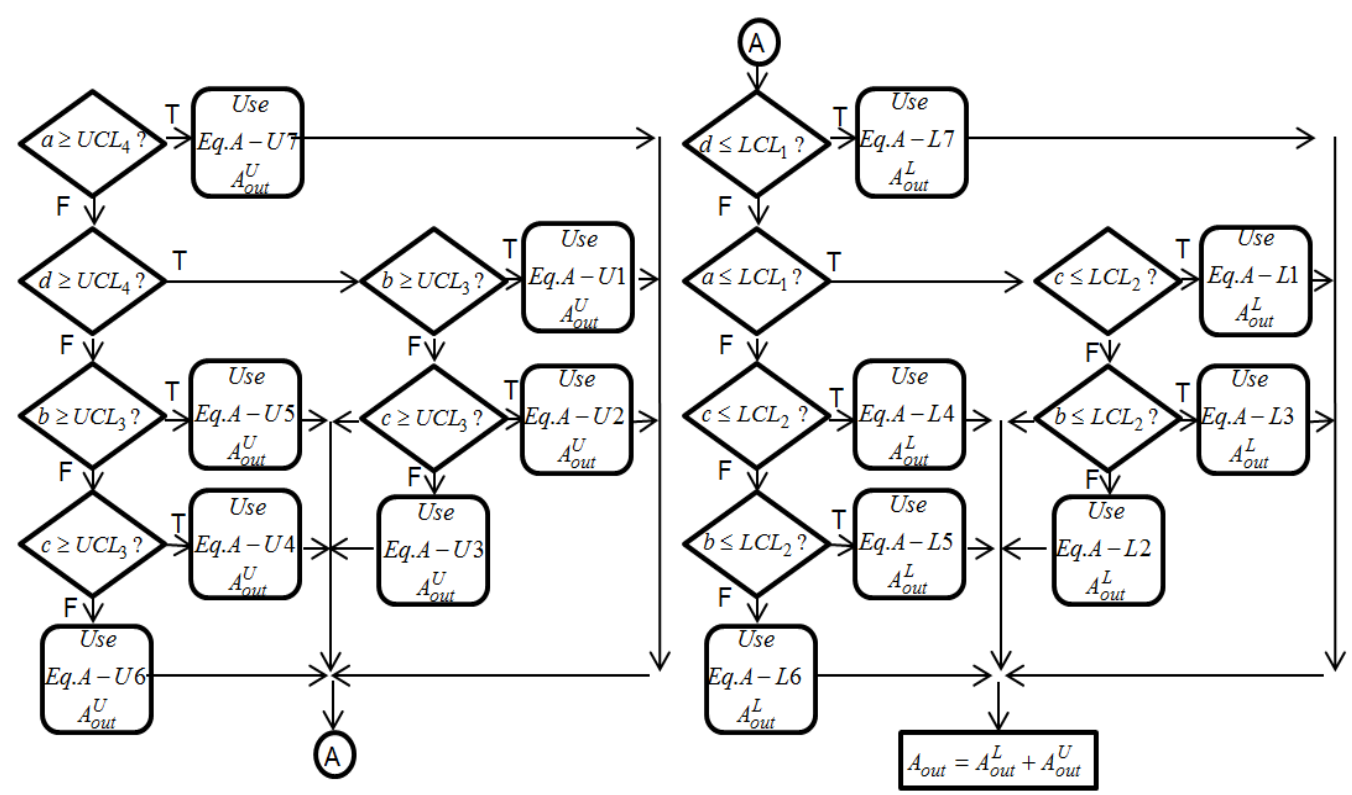

(Véase el anexo para las ecuaciones)

Fuente: basado en Gülbay y Kahraman (2007 y 2006) 


\section{REGLAS DE EVALUACIÓN}

Para evaluar el estado de control de la muestra obtenida en el proceso, autores como Montgomery (2004) y Gülbay y Kahraman (2006) recopilan y presentan reglas que determinan la normalidad o no normalidad de los datos, que sirven para detectar patrones no aleatorios o comportamientos no naturales. Las reglas utilizadas en este documento son las de Western Electric, Grant y Leavenworth, y las de Nelson. En la figura 3 se presenta la división de las zonas de un gráfico de control tres sigma, lo cual permite entender las reglas de evaluación para los gráficos de control tradicionales y difusos.

En la tabla 2 se presentan las reglas de evaluación de los gráficos de control tradicionales que determinan cuándo un gráfico se encuentra fuera de control. Por su parte, las reglas de evaluación para el análisis de los gráficos de control difusos se han planteado a partir de la conversión de las reglas desarrolladas para
Figura 3. Zonas de los gráficos de control

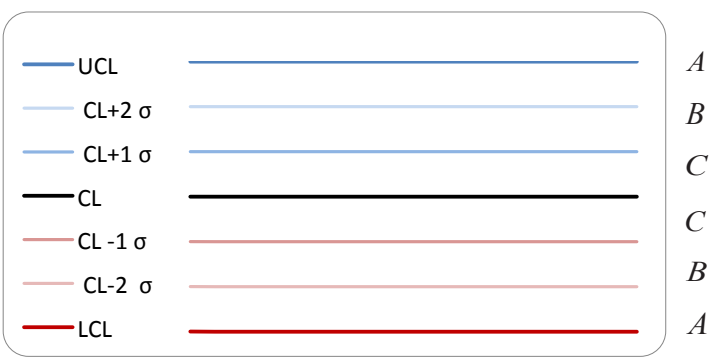

Fuente: Basado en Zarandi y Alaeddini (2010) y Gülbay y Kahraman (2006)

los gráficos de control tradicionales según Gülbay y Kahraman (2006), y se presentan en la tabla 3. Se debe tener en cuenta que las reglas de Nelson para los gráficos difusos son las mismas que para los tradicionales, excepto por las reglas 3 y 4 .

Tabla 2. Reglas de evaluación para los gráficos de control tradicionales

\begin{tabular}{|c|c|c|c|}
\hline Regla & Western Electric & Grant y Leavenworth & Nelson \\
\hline 1 & $\begin{array}{l}\text { Un punto se localiza fuera } \\
\text { de los límites de control tres } \\
\text { sigma }\end{array}$ & $\begin{array}{c}\text { Siete puntos consecutivos } \\
\text { en el mismo lado de la línea } \\
\text { central }(\mathrm{CL})\end{array}$ & $\begin{array}{l}\text { Uno o más puntos fuera de los límites de } \\
\text { control tres sigma }\end{array}$ \\
\hline 2 & $\begin{array}{c}\text { Dos de tres puntos } \\
\text { consecutivos caen fuera de } \\
\text { los límites dos sigma }\end{array}$ & $\begin{array}{c}\text { Por lo menos } 10 \text { de } 11 \text { puntos } \\
\text { consecutivos en el mismo lado } \\
\text { de } C L\end{array}$ & $\begin{array}{l}\text { Nueve puntos consecutivos en el mismo } \\
\text { lado de la línea central }\end{array}$ \\
\hline 3 & $\begin{array}{c}\text { Cuatro de cinco puntos } \\
\text { consecutivos caen a una } \\
\text { distancia de un sigma o más } \\
\text { de la línea central }\end{array}$ & $\begin{array}{l}\text { Por lo menos } 12 \text { de } 14 \text { puntos } \\
\text { consecutivos en el mismo lado } \\
\text { de } C L\end{array}$ & $\begin{array}{l}\text { Seis puntos consecutivos que incrementan o } \\
\text { decrecen constantemente }\end{array}$ \\
\hline 4 & $\begin{array}{l}\text { Ocho puntos consecutivos } \\
\text { caen en el mismo lado de } \mathrm{CL}\end{array}$ & $\begin{array}{c}\text { Por lo menos } 14 \text { de } 17 \text { puntos } \\
\text { consecutivos en el mismo lado } \\
\text { de } C L\end{array}$ & $\begin{array}{l}\text { Catorce puntos consecutivos alterando } \\
\text { arriba y abajo }\end{array}$ \\
\hline 5 & - & - & $\begin{array}{l}\text { Dos de tres puntos consecutivos en la zona } \\
\text { A o más allá }\end{array}$ \\
\hline 6 & - & - & $\begin{array}{l}\text { Cuatro de cinco puntos consecutivos en la } \\
\text { zona B o más allá }\end{array}$ \\
\hline 7 & - & - & $\begin{array}{l}\text { Quince puntos consecutivos en las zonas } C \text {, } \\
\text { arriba y debajo de } C L\end{array}$ \\
\hline 8 & - & - & $\begin{array}{l}\text { Ocho puntos consecutivos sobre ambos } \\
\text { lados de la línea central con ninguno en la } \\
\text { zona C }\end{array}$ \\
\hline
\end{tabular}

Fuente: basada en Montgomery (2004) y Gülbay y Kahraman (2006) 
Tabla 3. Reglas de evaluación para los gráficos de control difusos

\begin{tabular}{|c|c|c|c|}
\hline Regla & Western Electric & Grant y Leavenworth & Nelson \\
\hline 1 & $\begin{array}{c}\text { Un dato difuso cae fuera de los } \\
\text { límites de control de tres sigma, } \\
\text { con una proporción mayor al } \\
\text { porcentaje predefinido } \beta \text { del } \\
\text { área de la muestra en el nivel } a \\
\text { deseado }\end{array}$ & $\begin{array}{c}\text { Un grado de pertenencia total } \\
\text { de alrededor de } 7 \text { de } 7 \text { puntos } \\
\text { consecutivos en el mismo lado } \\
\text { de la línea central }\end{array}$ & \\
\hline 2 & $\begin{array}{c}\text { Un grado de pertenencia total } \\
\text { de alrededor de } 2 \text { de } 3 \text { puntos } \\
\text { consecutivos en la zona A o más } \\
\text { allá }\end{array}$ & $\begin{array}{c}\text { Al menos un grado de } \\
\text { pertenencia total, de } \\
\text { alrededor de } 10 \text { de } 11 \text { puntos } \\
\text { consecutivos en el mismo lado } \\
\text { de la línea central }\end{array}$ & \\
\hline 3 & $\begin{array}{c}\text { Un grado de pertenencia total } \\
\text { de alrededor de } 4 \text { de } 5 \text { puntos } \\
\text { consecutivos en la zona C o más } \\
\text { allá }\end{array}$ & $\begin{array}{c}\text { Al menos un grado de } \\
\text { pertenencia, del total de } \\
\text { alrededor de } 12 \text { de } 14 \text { puntos } \\
\text { consecutivos en el mismo lado } \\
\text { de la línea central }\end{array}$ & $\begin{array}{l}\text { Seis puntos seguidos } \\
\text { aumentando constantemente o } \\
\text { disminuyendo con respecto al } \\
\text { nivel } a \text { de rango medio deseado }\end{array}$ \\
\hline 4 & $\begin{array}{l}\text { Un grado de pertenencia total } \\
\text { de alrededor de } 8 \text { de } 8 \text { puntos } \\
\text { consecutivos en el mismo lado } \\
\text { de la línea central }\end{array}$ & $\begin{array}{c}\text { Por lo menos un grado } \\
\text { de pertenencia total de } \\
\text { alrededor de } 14 \text { de } 17 \text { puntos } \\
\text { consecutivos en el mismo lado } \\
\text { de la línea central }\end{array}$ & $\begin{array}{l}\text { Catorce puntos consecutivos } \\
\text { alterando arriba y abajo con } \\
\text { respecto al rango medio difuso } \\
\text { de nivel } a \text { deseado }\end{array}$ \\
\hline
\end{tabular}

Fuente: basada en Gülbay y Kahraman (2006)

\section{CASO DE ESTUDIO}

El caso de estudio se desarrolló mediante datos tomados del ejemplo 6.3 propuesto en Montgomery (2004). Este presenta el número de disconformidades observadas en 26 muestras sucesivas de 100 tarjetas de circuitos impresos, que se muestran en la tabla 4.

Tabla 4. Número de disconformidades por muestra

\begin{tabular}{|c|c|c|c|}
\hline N. ${ }^{\circ}$ de muestra & N. ${ }^{0}$ de disconformidades & N. ${ }^{0}$ de muestra & N. ${ }^{\circ}$ de disconformidades \\
\hline 1 & 21 & 14 & 19 \\
\hline 2 & 24 & 15 & 10 \\
\hline 3 & 16 & 16 & 17 \\
\hline 4 & 12 & 17 & 13 \\
\hline 5 & 15 & 18 & 22 \\
\hline 6 & 5 & 19 & 18 \\
\hline 7 & 28 & 20 & 39 \\
\hline 8 & 20 & 21 & 30 \\
\hline 9 & 31 & 22 & 24 \\
\hline 10 & 25 & 23 & 16 \\
\hline 11 & 20 & 24 & 19 \\
\hline 12 & 24 & 25 & 17 \\
\hline 13 & 16 & 26 & 15 \\
\hline
\end{tabular}

Fuente: Montgomery (2004) 


\section{GRÁFICO DE CONTROL C TRADICIONAL}

Se halló la media y la desviación estándar para seguir con el cálculo de los límites de control, y se obtuvo lo siguiente: $C L_{c}=19.84{ }_{U C L_{\mathrm{c}}}=33.21 \mathrm{y}_{L C L_{\mathrm{c}}}=6.48$

\section{GRÁFICO DE CONTROL C DIFUSO}

En el caso del gráfico de control $c$ difuso, cada muestra se representa por un número difuso trapezoidal, donde $b=c$. Se asumió un spread d = 2 (tabla 5), un valor de $\alpha=0.6$ y $\beta=0.7$. Luego se calculó la línea central y los límites de control difusos según las ecuaciones (1-3), cuyos resultados se presentan en la tabla 6 .

Posteriormente se calculó la línea central y los límites de control difusos con el uso de los métodos de transformación difusos: rango medio difuso de nivel $\alpha$, moda difusa y mediana difusa de nivel $\alpha$, presentados en la tabla 7. Se obtuvieron los mismos datos con los tres métodos aplicados.

Tabla 5. Número difuso trapezoidal para cada muestra

\begin{tabular}{cccccccccc}
\hline $\mathbf{N .}^{\mathbf{0}}$ & $\mathbf{a}$ & $\mathbf{b}$ & $\mathbf{c}$ & $\mathbf{d}$ & $\mathbf{N} \mathbf{~}^{{ }^{*}}$ & $\mathbf{a}$ & $\mathbf{b}$ & $\mathbf{c}$ & $\mathbf{d}$ \\
\hline 1 & 19 & 21 & 21 & 23 & 14 & 17 & 19 & 19 & 21 \\
\hline 2 & 22 & 24 & 24 & 26 & 15 & 8 & 10 & 10 & 12 \\
\hline 3 & 14 & 16 & 16 & 18 & 16 & 15 & 17 & 17 & 19 \\
\hline 4 & 10 & 12 & 12 & 14 & 17 & 11 & 13 & 13 & 15 \\
\hline 5 & 13 & 15 & 15 & 17 & 18 & 20 & 22 & 22 & 24 \\
\hline 6 & 3 & 5 & 5 & 7 & 19 & 16 & 18 & 18 & 20 \\
\hline 7 & 26 & 28 & 28 & 30 & 20 & 37 & 39 & 39 & 41 \\
\hline 8 & 18 & 20 & 20 & 22 & 21 & 28 & 30 & 30 & 32 \\
\hline 9 & 29 & 31 & 31 & 33 & 22 & 22 & 24 & 24 & 26 \\
\hline 10 & 23 & 25 & 25 & 27 & 23 & 14 & 16 & 16 & 18 \\
\hline 11 & 18 & 20 & 20 & 22 & 24 & 17 & 19 & 19 & 21 \\
\hline 12 & 22 & 24 & 24 & 26 & 25 & 15 & 17 & 17 & 19 \\
\hline 13 & 14 & 16 & 16 & 18 & 26 & 13 & 15 & 15 & 17 \\
\hline
\end{tabular}

Fuente: elaboración propia

Tabla 6. Límites de control del gráfico de control c difuso

\begin{tabular}{ccccccccccccc}
\hline \multicolumn{1}{c}{$\widetilde{C} L_{\tilde{c}}$} & \multicolumn{1}{c}{$L \widetilde{C} L_{\widetilde{c}}$} & \multicolumn{4}{c}{$U \widetilde{C} L_{\widetilde{c}}$} & \\
\hline$C L_{1}$ & $C L_{2}$ & $C L_{3}$ & $C L_{4}$ & $C L_{1}$ & $C L_{2}$ & $C L_{3}$ & $C L_{4}$ & $C L_{1}$ & $C L_{2}$ & $C L_{3}$ & $C L_{4}$ \\
\hline 17.85 & 19.85 & 19.85 & 21.85 & 3.82 & 6.48 & 6.48 & 9.17 & 30.52 & 33.21 & 33.21 & 35.87 \\
\hline
\end{tabular}


Tabla 7. Límites de control usando rango medio, moda y mediana difusos

\begin{tabular}{|c|c|c|c|c|c|}
\hline$C L_{m r}^{a}$ & $C L_{\text {mod }}$ & $C L_{\text {med }}^{a} L C L_{m r}^{a}$ & $L C L_{\text {mod }} L C L_{\text {med }}^{a}$ & $U C L_{m r}^{a}$ & $U C L_{\text {mod }} U C L_{\text {med }}^{a}$ \\
\hline & 19.85 & & 6.48 & & 33.21 \\
\hline
\end{tabular}

Fuente: elaboración propia

\section{ANÁLISIS DEL GRÁFICO DE CONTROL C}

Al analizar el gráfico c tradicional con las reglas de Western Electric se puede observar que este cumple con la regla 1 , pues presenta dos datos por fuera

Figura 4. Gráfico c tradicional

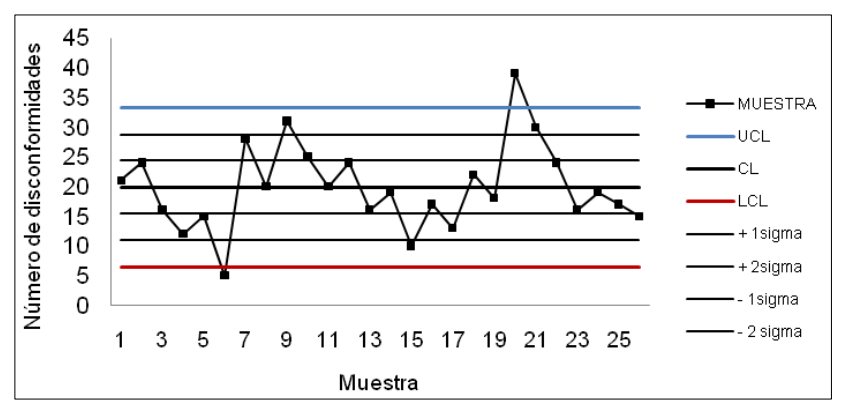

de los límites de control $3 \sigma$ (figura 4), lo que implica que el proceso presenta patrones de comportamiento atribuibles a alguna causa no natural.

El gráfico de control no cumple con ninguna de las reglas de Leavenworth. Al evaluar este gráfico con las reglas de Nelson, se nota que cumple con la regla número 1 , ratificando lo encontrado con las reglas de Western Electric.

Por otro lado, al analizar el gráfico de control c difuso utilizando la moda difusa, rango medio difuso y mediana difusa se obtuvieron los resultados presentados en la tabla 8. Como se puede observar, los tres métodos de transformación coinciden en que las muestras 6 y 20 se encuentran fuera de control.

Tabla 8. Decisiones de control según la moda, rango medio y mediana $(a=0.60, \beta=0.70)$

\begin{tabular}{|c|c|c|c|c|c|c|c|}
\hline $\begin{array}{l}\mathrm{N} .{ }^{\circ} \text { de } \\
\text { muestra }\end{array}$ & $b_{j}=c_{j}$ & $\beta_{j}$ & Moda & $f_{m r, j}^{\alpha=0,6}$ & Rango medio & $f_{\text {med }, j}^{\alpha=0,6}$ & Mediana \\
\hline 1 & 21 & 100 & En control & 21.00 & En control & 21.00 & En control \\
\hline 2 & 24 & 1.00 & En control & 24.00 & En control & 24.00 & En control \\
\hline 3 & 16 & 1.00 & En control & 16.00 & En control & 16.00 & En control \\
\hline 4 & 12 & 1.00 & En control & 12.00 & En control & 12.00 & En control \\
\hline 5 & 15 & 1.00 & En control & 15.00 & En control & 15.00 & En control \\
\hline 6 & 5 & 0.00 & Fuera de control & 5.00 & Fuera de control & 5.00 & Fuera de control \\
\hline 7 & 28 & 1.00 & En control & 28.00 & En control & 28.00 & En control \\
\hline 8 & 20 & 1.00 & En control & 20.00 & En control & 20.00 & En control \\
\hline 9 & 31 & 1.00 & En control & 31.00 & En control & 31.00 & En control \\
\hline 10 & 25 & 1.00 & En control & 25.00 & En control & 25.00 & En control \\
\hline 11 & 20 & 1.00 & En control & 20.00 & En control & 20.00 & En control \\
\hline 12 & 24 & 1.00 & En control & 24.00 & En control & 24.00 & En control \\
\hline 13 & 16 & 1.00 & En control & 16.00 & En control & 16.00 & En control \\
\hline 14 & 19 & 1.00 & En control & 19.00 & En control & 19.00 & En control \\
\hline 15 & 10 & 1.00 & En control & 10.00 & En control & 10.00 & En control \\
\hline 16 & 17 & 1.00 & En control & 17.00 & En control & 17.00 & En control \\
\hline 17 & 13 & 1.00 & En control & 13.00 & En control & 13.00 & En control \\
\hline 18 & 22 & 1.00 & En control & 22.00 & En control & 22.00 & En control \\
\hline
\end{tabular}




\begin{tabular}{cccccccc}
\hline $\begin{array}{c}\text { N. }{ }^{\circ} \text { de } \\
\text { muestra }\end{array}$ & $b_{j}=c_{j}$ & $\beta_{j}$ & Moda & $f_{m r, j}^{\alpha=0,6}$ & Rango medio & $f_{m e d, j}^{\alpha=0,6}$ & Mediana \\
\hline 19 & 18 & 1.00 & En control & 18.00 & En control & 18.00 & En control \\
\hline 20 & 39 & 0.00 & Fuera de control & 39.00 & Fuera de control & 39.00 & Fuera de control \\
\hline 21 & 30 & 1.00 & En control & 30.00 & En control & 30.00 & En control \\
\hline 22 & 24 & 1.00 & En control & 24.00 & En control & 24.00 & En control \\
\hline 23 & 16 & 1.00 & En control & 16.00 & En control & 16.00 & En control \\
\hline 24 & 19 & 1.00 & En control & 19.00 & En control & 19.00 & En control \\
\hline 25 & 17 & 1.00 & En control & 17.00 & En control & 17.00 & En control \\
\hline 26 & 15 & 1.00 & En control & 15.00 & En control & 15.00 & En control \\
\hline
\end{tabular}

Fuente: elaboración propia

Mediante el método del enfoque difuso directo se calculó el área de la muestra que se encuentra por fuera de los límites de control $A_{\text {out }}$, y luego se calculó el porcentaje del área de la muestra dentro de los límites de control $\beta_{j}^{\alpha}$. Para esto se utilizó el algoritmo presentado en la figura 2 y la expresión del método DFA para evaluar la condición de control de cada muestra. Los resultados se presentan en la tabla 9 . Como se puede observar, según este método, las muestras 6 y 20 se encuentran fuera de control.

Con el uso del DFA se aplicaron las reglas de evaluación para gráficos difusos. Inicialmente se calcularon las zonas difusas (tabla 10), y luego se calculó la pertenencia de cada muestra en las diferentes zonas; en la tabla 11 se presenta la pertenencia a las zonas $+3 \sigma$ hasta $\mathrm{CL}$ de las muestras 19 a la 26; la muestra 20 tiene un grado de pertenencia igual a 1 en la zonas $\mathrm{E}+3 \sigma$, lo que indica que el área de esta muestra está completamente fuera del límite. De esta manera, se encontró la pertenencia de cada una de las muestras a las reglas de evaluación de los gráficos difusos.
La evaluación de las reglas de Western Electric presentó que solo se cumple la regla 1, por lo menos 1 punto por fuera de los límites $3 \sigma$, y la evaluación de las reglas de Grant y Leavenworth deja en claro que las muestras no cumplen con ninguna regla, pues el grado de pertenencia es menor a 1. De acuerdo con las reglas de Nelson, se cumple la regla 1, una muestra fuera de los límites de control.

Después de realizar el análisis de cada uno de los gráficos se puede decir que todos coinciden en sus resultados, dejando en claro que las muestras 6 y 20 están fuera de control porque están situadas fuera de los límites; además, no existe ningún otro tipo de comportamiento anormal. Esta condición de fuera de control se debe a causas asignables, que en este caso se atribuyen a la falta de precisión en el reconocimiento de los tipos de disconformidades que pudieron estar presentes y a un problema en la temperatura de la máquina de la ola de soldadura, que se reparó subsecuentemente, para cada una de las muestras, respectivamente (Gülbay y Kahraman, 2006).

Tabla 9. Decisión de control basada en el DFA

\begin{tabular}{cccccccccccccc}
\hline $\begin{array}{c}\text { N. }{ }^{\circ} \mathrm{de} \\
\text { Muestra }\end{array}$ & $A_{\text {out }}$ & $A_{\text {out }}^{U}$ & $A_{\text {out }}^{L}$ & $\begin{array}{c}\text { Área de } \\
\text { Muestra }\end{array}$ & $\beta_{j}$ & Decisión DFA & $\begin{array}{c}\text { N. }{ }^{\circ} \text { de } \\
\text { Muestra }\end{array}$ & $A_{\text {out }}$ & $A_{\text {out }}^{U}$ & $A_{\text {out }}^{L}$ & $\begin{array}{c}\text { Área de } \\
\text { Muestra }\end{array}$ & $\beta_{j}$ Decisión DFA \\
\hline 1 & 0.00 & 0.00 & 0.00 & 0.32 & 1 & En control & 14 & 0.00 & 0.00 & 0.00 & 0.32 & 1 & En control \\
\hline 2 & 0.00 & 0.00 & 0.00 & 0.32 & 1 & En control & 15 & 0.00 & 0.00 & 0.00 & 0.32 & 1 & En control \\
\hline 3 & 0.00 & 0.00 & 0.00 & 0.32 & 1 & En control & 16 & 0.00 & 0.00 & 0.00 & 0.32 & 1 & En control \\
\hline 4 & 0.00 & 0.00 & 0.00 & 0.32 & 1 & En control & 17 & 0.00 & 0.00 & 0.00 & 0.32 & 1 & En control \\
\hline 5 & 0.00 & 0.00 & 0.00 & 0.32 & 1 & En control & 18 & 0.00 & 0.00 & 0.00 & 0.32 & 1 & En control \\
\hline 6 & 0.30 & 0.00 & 0.30 & 0.32 & 0.049 & Fuera de control & 19 & 0.00 & 0.00 & 0.00 & 0.32 & 1 & En control \\
\hline 7 & 0.00 & 0.00 & 0.00 & 0.32 & 1 & En control & 20 & 0.32 & 0.32 & 0.00 & 0.32 & - & $\begin{array}{c}\text { Fuera de } \\
\text { control }\end{array}$ \\
\hline 8 & 0.00 & 0.00 & 0.00 & 0.32 & 1 & En control & 21 & 0.00 & 0.00 & 0.00 & 0.32 & 1 & En control \\
\hline
\end{tabular}




\begin{tabular}{cccccccccccccc}
\hline $\begin{array}{c}\text { N. }{ }^{\circ} \mathrm{de} \\
\text { Muestra }\end{array}$ & $A_{\text {out }}$ & $A_{\text {out }}^{U}$ & $A_{\text {out }}^{L}$ & $\begin{array}{c}\text { Área de } \\
\text { Muestra }\end{array}$ & & Decisión DFA & $\begin{array}{c}\text { N. }{ }^{\circ} \text { de } \\
\text { Muestra }\end{array}$ & $A_{\text {out }}$ & $A_{\text {out }}^{U}$ & $A_{\text {out }}^{L}$ & $\begin{array}{c}\text { Área de } \\
\text { Muestra }\end{array}$ & Decisión DFA \\
\hline 9 & 0.00 & 0.00 & 0.00 & 0.32 & 1 & En control & 22 & 0.00 & 0.00 & 0.00 & 0.32 & 1 & En control \\
\hline 10 & 0.00 & 0.00 & 0.00 & 0.32 & 1 & En control & 23 & 0.00 & 0.00 & 0.00 & 0.32 & 1 & En control \\
\hline 11 & 0.00 & 0.00 & 0.00 & 0.32 & 1 & En control & 24 & 0.00 & 0.00 & 0.00 & 0.32 & 1 & En control \\
\hline 12 & 0.00 & 0.00 & 0.00 & 0.32 & 1 & En control & 25 & 0.00 & 0.00 & 0.00 & 0.32 & 1 & En control \\
\hline 13 & 0.00 & 0.00 & 0.00 & 0.32 & 1 & En control & 26 & 0.00 & 0.00 & 0.00 & 0.32 & 1 & En control \\
\hline
\end{tabular}

Fuente: elaboración propia

Tabla 10. Límites de control para las zonas difusas

\begin{tabular}{cccccccc}
\hline $\mathbf{N .}^{{ }^{\boldsymbol{}}}$ & $\mathbf{U C L}$ & $\mathbf{2}^{\boldsymbol{\sigma}}$ & $\mathbf{1}^{\boldsymbol{\sigma}}$ & $\mathbf{C L}$ & $\mathbf{- 1}^{\boldsymbol{\sigma}}$ & $\mathbf{- 2} \boldsymbol{\sigma}$ & $\mathbf{L C L}$ \\
\hline $\mathrm{a}$ & 30.52 & 26.3 & 22.07 & 17.85 & 13.17 & 8.5 & 3.82 \\
\hline $\mathrm{b}$ & 33.21 & 28.76 & 24.3 & 19.85 & 15.39 & 10.94 & 6.48 \\
\hline $\mathrm{c}$ & 33.21 & 28.76 & 24.3 & 19.85 & 15.39 & 10.94 & 6.48 \\
\hline $\mathrm{d}$ & 35.87 & 31.19 & 26.52 & 21.85 & 17.62 & 13.4 & 9.17 \\
\hline
\end{tabular}

Fuente: elaboración propia

Tabla 11. Pertenencia de las muestras difusas a las zonas $+3 \sigma$ hasta CL (E: encima; En: dentro; D: debajo)

\begin{tabular}{|c|c|c|c|c|c|c|c|c|c|c|c|c|}
\hline N. ${ }^{o}$ & $\mathrm{E}+3^{\sigma}$ & $\mathrm{En}+3^{\sigma}$ & $\mathrm{D}+3^{\sigma}$ & $\mathrm{E}+2^{\sigma}$ & $\mathrm{En}+2 \sigma$ & $\mathrm{D}+2^{\sigma}$ & $\mathrm{E}+1 \sigma$ & $\mathrm{En}+1 \mathrm{v}$ & $\mathrm{D}+1^{\sigma}$ & E $C L$ & En $\mathrm{C} L$ & $\mathrm{DCL}$ \\
\hline 19 & 0.00 & 0.00 & 1.00 & 0.00 & 0.00 & 1.00 & 0.00 & 0.00 & 1.00 & 0.00 & 0.00 & 1.00 \\
\hline 20 & 1.00 & 0.00 & 0.00 & 1.00 & 0.00 & 0.00 & 1.00 & 0.00 & 0.00 & 1.00 & 0.00 & 0.00 \\
\hline 21 & 0.00 & 0.00 & 1.00 & 0.90 & 0.10 & 0.00 & 1.00 & 0.00 & 0.00 & 1.00 & 0.00 & 0.00 \\
\hline 22 & 0.00 & 0.00 & 1.00 & 0.00 & 0.00 & 1.00 & 0.00 & 0.71 & 0.29 & 1.00 & 0.00 & 0.00 \\
\hline 23 & 0.00 & 0.00 & 1.00 & 0.00 & 0.00 & 1.00 & 0.00 & 0.00 & 1.00 & 0.00 & 0.00 & 1.00 \\
\hline 24 & 0.00 & 0.00 & 1.00 & 0.00 & 0.00 & 1.00 & 0.00 & 0.00 & 1.00 & 0.00 & 0.22 & 0.78 \\
\hline 25 & 0.00 & 0.00 & 1.00 & 0.00 & 0.00 & 1.00 & 0.00 & 0.00 & 1.00 & 0.00 & 0.00 & 1.00 \\
\hline
\end{tabular}

Fuente: elaboración propia

\section{CONCLUSIONES}

Cuando se compararon los gráficos de control c tradicionales con los gráficos de control c difusos, considerando patrones de comportamiento no naturales, se encontró que ambos tipos de gráficos identifican de manera concluyente los comportamientos anormales del proceso.

Según la investigación, con base en los datos utilizados, no se encuentran diferencias en los resultados de evaluación obtenidos con los gráficos de control tradiciones y los gráficos de control difusos. De esta manera, se puede decir que el gráfico de control c tradicional representa una herramienta que requiere un menor esfuerzo en el momento de establecer un control de proceso y obtiene resultados similares que el gráfico de control c difuso.
En el momento de evaluar los gráficos de control c difusos con las reglas de Western Electric y Grant, y Leavenworth, se identificó que con el cálculo del área de cada muestra se puede determinar su estado de control, y, además, aplicar las reglas de evaluación de los gráficos que determinan patrones de comportamiento no aleatorio.

Aunque los gráficos de control c tradicionales representan una buena alternativa, la opción de incluir la lógica difusa en la construcción de los gráficos permite el manejo de la imprecisión en los datos y la construcción de un gráfico de control c que refleje apropiadamente los comportamientos no aleatorios del proceso, información que no maneja un gráfico c tradicional. 
Si bien los resultados de los gráficos c difusos son similares a los del gráfico tradicional, en el momento que se tenga la información imprecisa y vaga se contará con una herramienta que brindará iguales elementos de juicio para la toma de decisiones.

\section{REFERENCIAS}

Alaeddini, A., Ghazanfari, M., y Nayeri, M. A. (2009). A hybrid fuzzy-statistical clustering approach for estimating the time of changes in fixed and variable sampling control charts. Information Sciences, 179(11), 1769-1784.

Amirzadeh, V., Mashinchi, M., y Parchami, A. (2009). Construction of p-charts using degree of nonconformity. Information Sciences, 179(1), 150160.

Briceño Beltrán, C. O. (2004). Redes neuronales artificiales, lógica difusa, geometría de fractales $\left(n .{ }^{\circ}\right.$ 006.3 B849r). Cali: Cenicaña.

Cheng, C. B. (2005). Fuzzy process control: Construction of control charts with fuzzy numbers. Fuzzy Sets and Systems, 154(2), 287-303.

Csutora, R., y Buckley, J. J. (2001). Fuzzy hierarchical analysis: The Lambda-Max method. Fuzzy Sets and Systems, 120(2), 181-195.

Demirli, K., y Vijayakumar, S. (2010). Fuzzy logic based assignable cause diagnosis using control chart patterns. Information Sciences, 180(17), 32583272.

Evans, J. R., y lindsay, W. M. (2005). Administración y control de la calidad (6. $\mathrm{a}$ ed.). México: Editorial Thomson. 516-524.

Faraz, A., y Shapiro, A. F. (2010). An application of fuzzy random variables to control charts. Fuzzy Sets and Systems, 161(20), 2684-2694.

Gryna, F. M., Chua, R. C., y Defeo, J. A. (2007). Método Juran: Análisis y planeación de la calidad. México: McGraw-Hill/Interamericana.

Gülbay, M., y Kahraman, C. (2006). Development of fuzzy process control charts and fuzzy unnatural pattern analyses. Computational Statistics \& Data Analysis, 51(1), 434-451.

Gülbay, M., y Kahraman, C. (2007). An alternative approach to fuzzy control charts: Direct fuzzy approach. Information Sciences, 177(6), 14631480.

Hryniewicz, O. (2007). Statistics with fuzzy data in statistical quality control. Soft Computing, 12(3), 229-234.

Hsieh, Yuh-Wen. (2005). On the construction of fuzzy control charts for variables (tesis de maestría). College of Management, Statistics Department. Taiwán. Disponible en: http://Etdncku.lib.ncku. edu.tw/etd-0628106-173512.pdf

Kaya, İ., y Kahraman, C. (2011a). Process capability analyses based on fuzzy measurements and fuzzy control charts. Expert Systems with Applications, 38(4), 3172-3184.

Kaya, I., yKahraman,C.(2011b). Fuzzy processcapability indices with asymmetric tolerances. Expert Systems with Applications, 38(12), 14882-14890.

Lazzari, L. L., Machado, E. A. M., y Pérez, R. H. (1998). Teoría de la decisión fuzzy. Buenos Aires: Macchi, 108.

Montgomery, D. (2004). Control estadístico de la calidad (3.a ed.). México: Limusa Wiley.

Noori, S., Bagherpour, M., y Zareei, A. (2008). Applying fuzzy control chart in earned value analysis: A new application. World Applied Sciences Journal, 3(4), 684-690.

Senturk, S., y Erginel, N. (2009). Development of fuzzy and control charts using $\alpha$-cuts. Information Sciences, 179(10), 1542-1551.

Shu, M. H., y Wu, H. (2011). Fuzzy X and R control charts: Fuzzy dominance approach. Computers \& Industrial Engineering, 61(3), 676-685.

Zarandi, M. H. F., y Alaeddini, A. (2010). A general fuzzy-statistical clustering approach for estimating the time of change in variable sampling control charts. Information Sciences, 180(16), 3033-3044. 
Anexo. Ecuaciones para calcular el área fuera de los límites de control de muestra ${ }^{1}$

A-U1

$A_{\text {out }}^{U}=\frac{\left\lfloor\left(d^{\alpha}-U C L_{4}^{\alpha}\right)+\left(d^{t}-U C L_{4}^{T}\right)\right\rfloor}{2}(\max (t-\alpha, 0))+\frac{\left\lfloor\left(d^{z}-a^{z}\right)+(c-b)\right\rfloor}{2}(\min (1-t, 1-\alpha))$

Donde $t=\left(U C L_{4}-a\right) /\left(\left(U C L_{4}-U C L_{3}\right)+(b-a)\right) \quad$ y $\quad z=\max (t, \alpha)$

$\mathrm{A}-\mathrm{U} 2$

$\left.\left.A_{\text {out }}^{U}=\left(\left[\left(d^{\alpha}-U C L_{4}^{\alpha}\right)+\left(c-U C L_{3}\right)\right)\right] 1-\alpha\right)\right) / 2$

A-U3

$A_{\text {out }}^{U}=\left(\left(d^{\alpha}-U C L_{4}^{\alpha}\right)(\max (t-\alpha, 0))\right) / 2$

Donde $t=\left(U C L_{4}-d\right) /\left(\left(U C L_{4}-U C L_{3}\right)-(D-C)\right)$

A-U4

$\left.\left.A_{\text {out }}^{U}=\left(\left(\left(c-U C L_{3}\right)+\left(d^{z}-U C L_{4}^{z}\right)\right)\right] \min (1-t, 1-\alpha)\right)\right) / 2$

Donde $t=\left(U C L_{4}-d\right) /\left(\left(U C L_{4}-U C L_{3}\right)-(d-c)\right) \quad$ y $\quad z=\max (t, \alpha)$

A-U5

$A_{\text {out }}^{U}=\frac{1}{2}\left[\left(d^{z 2}-U C L_{4}^{Z 2}\right)+\left(d^{t 1}-U C L_{4}^{t 1}\right)\right]\left(\min \left(\max \left(t_{1}-\alpha, 0\right), t_{1}-t_{2}\right)\right)$

$+\frac{1}{2}\left[\left(d^{z 1}-a^{Z 1}\right)+(c-b)\right]\left(\min \left(1-t_{1}, 1-\alpha\right)\right)$

Donde $\begin{aligned} t & =\frac{U C L_{4}-a}{(b-a)+\left(U C L_{4}-U C L_{3}\right)} \quad \mathrm{t}_{2}=\frac{U C L_{4}-d}{\left(U C L_{4}-U C L_{3}\right)-(d-c)} \\ z_{1} & =\max \left(\alpha-t_{1}\right) \quad \mathrm{y} \quad \mathrm{z}_{2}=\max \left(\alpha, t_{2}\right)\end{aligned}$

A-U6 $A_{\text {out }}^{U}=0$

A-U7 $\quad A_{\text {out }}^{U}=\frac{1}{2}\left[\left(d^{\alpha}-a^{\alpha}\right)+(c-b)\right](1-\alpha)$

A-L1 $A_{\text {out }}^{U}=\frac{1}{2}\left[\left(L C L_{1}^{\alpha}-a^{\alpha}\right)+\left(L C L_{1}^{t}-a^{t}\right)\right](\max (t-\alpha, 0))$

$+\frac{1}{2}\left[\left(d^{z}-a^{z}\right)+(c-b)\right] \min (1-t, 1-\alpha)$

Donde $_{t}=\left(d-L C L_{1}\right) /\left(\left(L C L_{2}-L C L_{1}\right)+(d-c)\right) \quad \mathrm{y} z=\max (\alpha, t)$

A-L2

$A_{\text {out }}^{L}=\frac{1}{2}\left[\left(d^{\alpha}-a^{\alpha}\right)+(c-b)\right](1-\alpha)$

A-L3

$A_{\text {out }}^{U}=\frac{1}{2}\left(d^{\alpha}-U C L_{4}^{\alpha}\right)(\max (t-\alpha, 0)$

A-L4

$\left.A_{\text {out }}^{L}=\frac{1}{2}\left[\left(L C L_{1}^{z 2}-a^{z 2}\right)+\left(L C L_{1}^{t 1}-a^{t 1}\right)\right] \min \left(\max \left(t_{1}-\alpha, 0\right), t_{1}-t_{2}\right)\right)$

$+\frac{1}{2}\left[\left(d^{z 1}-a^{Z 1}\right)+(c-b)\right]\left(\min \left(1-t_{1}, 1-\alpha\right)\right)$

Donde

$$
t_{1}=\frac{d-L C L_{1}}{\left(L C L_{2}-L C L_{1}\right)+(d-c)} \quad \mathrm{t}_{2}=\frac{a-L C L_{1}}{\left(L C L_{2}-L C L_{1}\right)-(b-a)}
$$

$$
z_{1}=\max \left(\alpha, t_{1}\right) \quad y \quad z_{2}=\max \left(\alpha, t_{2}\right)
$$

A-L5

$$
A_{\text {out }}^{L}=\frac{1}{2}\left[\left(L C L_{1}^{z}-a^{z}\right)+\left(L C L_{2}-b\right)\right](\min (1-t, 1-\alpha))
$$

Donde $t_{1}=\frac{a-L C L_{1}}{\left(L C L_{2}-L C L_{1}\right)-(b-a)} \quad$ y $\quad \mathrm{z}=\max (\alpha, \mathrm{t})$

A-L6 $A_{\text {out }}^{L}=0$

A-L7 $A_{\text {out }}^{L}=\frac{1}{2}\left[\left(d^{\alpha}-a^{\alpha}\right)+(c-b)\right](1-\alpha)$

Fuente: adaptado de Gülbay and Kahraman (2006 y 2007)

1 Cuando se realizó la revisión bibliográfica y el análisis de estas ecuaciones se encontró que el autor había cometido un error en una ecuación, en t de A-U2. 